\title{
EFFETS DE LA DENSITE DE POPULATION ET DU GROUPEMENT SUR LA REPRODUCTION \\ DE BRACHIONUS CALYCIFLORUS (PALLAS) \\ [ROTIFERE]
}

\author{
par R. POURRIOT ${ }^{1}$ et C. ROUGIER ${ }^{1}$
}

\begin{abstract}
Une influence complexe de la densité de population et, surtout, du groupement est mise en évidence sur un clone de $B$. calyciflorus. A deux densités de population $(0,2$ et 2$)$ sur les quatre testées $(0,2-0,6-1-2)$, le taux de femelles mictiques en $F_{1}$ est augmenté lorsque les femelles parentales sont groupées par deux et diminué lorsqu'elles sont groupées par six. Le groupement par 6 exerce également un effet négatif sur la fécondité. Ces effets sont difficilement interprétables et diffèrent de ceux obtenus par Gilbert (1963) sur une souche de la même espèce.
\end{abstract}

\section{Effects of population density and grouping on the reproduction of Brachionus calyciflorus (Pallas).}

The complex influence of population density, and especially grouping, is shown by a clone of $B$. calciflorus. Four population densities $(0.2,0.6,1,2)$ were used in the experiments, and at two densities $(0.2,2)$ the production of miotic females in $F_{1}$ is higher when the parent females are grouped in twos, and lower when they are grouped in sixes. The grouping in sizes also has a negative effect on the fecundity. These effects are difficult to interpret and are different from the results obtained by Gilbert (1963) who worked on a strain of the same species.

Nous avons récemment montré que le taux de femelles mictiques dans la descendance $F_{1}$ d'une femelle de $B$. calyciflorus dépendait de l'âge de celle-ci (Pourriot et Rougier, 1976² ; Rougier and Pourriot 1977). Or Gilbert (1963) a mis en évidence chez cette espèce, une influence déterminante de la densité de population sur la production de femelles mictiques. Il était donc intéressant de tester les réactions de la souche précédemment expérimentée vis-à-vis de ce facteur, déjà reconnu par Buchner $(1941,1969)$ comme efficace pour accroître le taux de mixis.

1. Laboratoire de Zoologie, Ecole Normale Supérieure, 46, rue d'Ulm, $75230 \mathrm{~Pa}$ ris Cedex 05.

2. Une erreur d'impression est cause de l'inversion des deux figures dans cette publication : la figure correspondant à $B$. calyciflorus a été attribuée à $B$. rubens et vice-versa. 


\section{1. - MATERIEL ET METHODES}

Ceux-ci, de même que la procédure expérimentale suivie ont été décrits dans un précédent travail (Rougier and Pourriot, $1977 \mathrm{~b}$ ). Nous les rappellerons brièvement :

— origine de la souche "GC». La souche a été constituée (comme toutes nos cultures de Rotifères) à partir d'une femelle isolée en mars 1974. Les œufs de durée produits dans cette culture n'ont jamais pu être induits à éclore. Il s'agit donc, en fait, d'un clone véritable puisque cette inaptitude à l'éclosion des œufs de durée assure que tous les descendants ont été obtenus parthénogénétiquement (parthénogenèse mitotique, cf. King, 1977) et sont donc, sauf accident, génétiquement semblables à la femelle initiale ;

- milieu de culture : eau de source non calcique (Volvic);

- nourriture: Phacus pyrum fournie en excès (une goutte, soit $1 / 20 \mathrm{ml}$, d'une culture dense par $\mathrm{ml}$ de milieu et par jour);

- température : $15^{\circ} \mathrm{C}$.

Quatre densités de population et trois groupements ont été étudiés : $0,2-0,6-1$ et $2 \% \% / \mathrm{ml}$, les femelles parentales étant soit isolées, soit groupées par 2 ou par 6 dès leur naissance et le début de l'expérience.

Toutes les expériences sont faites en milieu confiné, c'est-à-dire non renouvelé pendant toute la durée de celles-ci.

Les analyses de variance sont toutes effectuées sur les pourcentages après transformation $\sin ^{-1} \vee \mathrm{p}$.

A $15^{\circ} \mathrm{C}$, il s'écoule environ 2 jours entre l'éclosion de la jeune femelle et la ponte de son premier œuf. Le développement embryonnaire de ce dernier s'effectue également en 2 jours. Cinq jours après le début des expériences, les jeunes correspondant au premier jour de ponte sont isolés. Les descendants suivants sont prélevés quotidiennement. Les expériences ont été arrêtées arbitrairement au

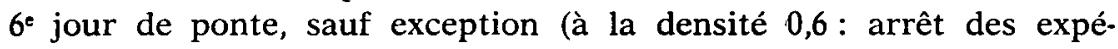
riences au $5^{\mathrm{e}}$ jour).

\section{2. - RESULTATS}

Nous disposons ainsi de pourcentages quotidiens de femelles mictiques et de pourcentages globaux correspondant à l'ensemble de la ponte obtenue en 5 ou 6 jours (tabl. 1 et fig. 1 à 4).

Il est a priori difficile de relever une variation des pourcentages globaux de femelles mictiques en fonction des densités de population expérimentées. Cependant, une analyse de variance à deux facteurs 
avec répétitions, effectuée sur les pourcentages globaux transformés $\left(\sin ^{-1} \vee \mathrm{p}\right)$ obtenus en 5 jours de ponte, montre que la densité de population a une influence significative $\left(\mathrm{F}=7,69\right.$ pour $\left.\mathrm{F}^{3}{ }_{108}=2,68\right)$, de même que le groupement $\left(F=13,12\right.$ pour $\left.\mathrm{F}^{2}{ }_{108}=3,07\right)$ et qu'il apparaît une interaction entre ces deux facteurs $\left(F=9,11\right.$ pour $\mathrm{F}^{6}{ }_{108}$ $=2,17)$.

\section{1. - Effets du groupement aux quatre densités de population}

Une seconde analyse de variance concernant l'influence du groupement et celle de l'âge des femelles parentales sur les pourcentages quotidiens (transformés $\sin ^{-1} \vee \mathrm{p}$ ) de femelles mictiques (tabl. 1) permet de clarifier ces résultats.

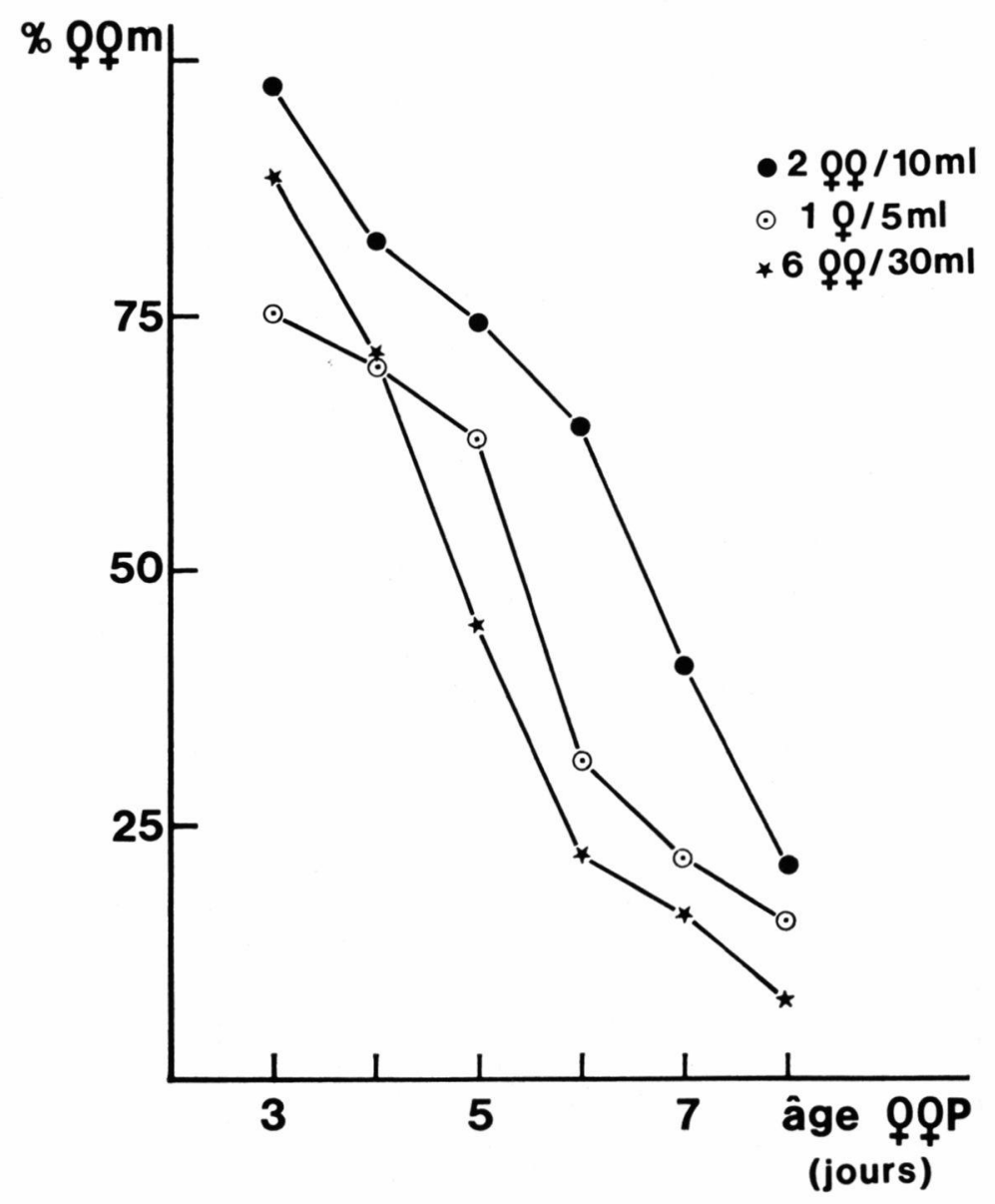

Fig. 1. - Evolution du taux de femelles mictiques en fonction de l'âge de la mère à la densité de population $0,2 \% / \mathrm{ml}$, pour trois groupements différents. 


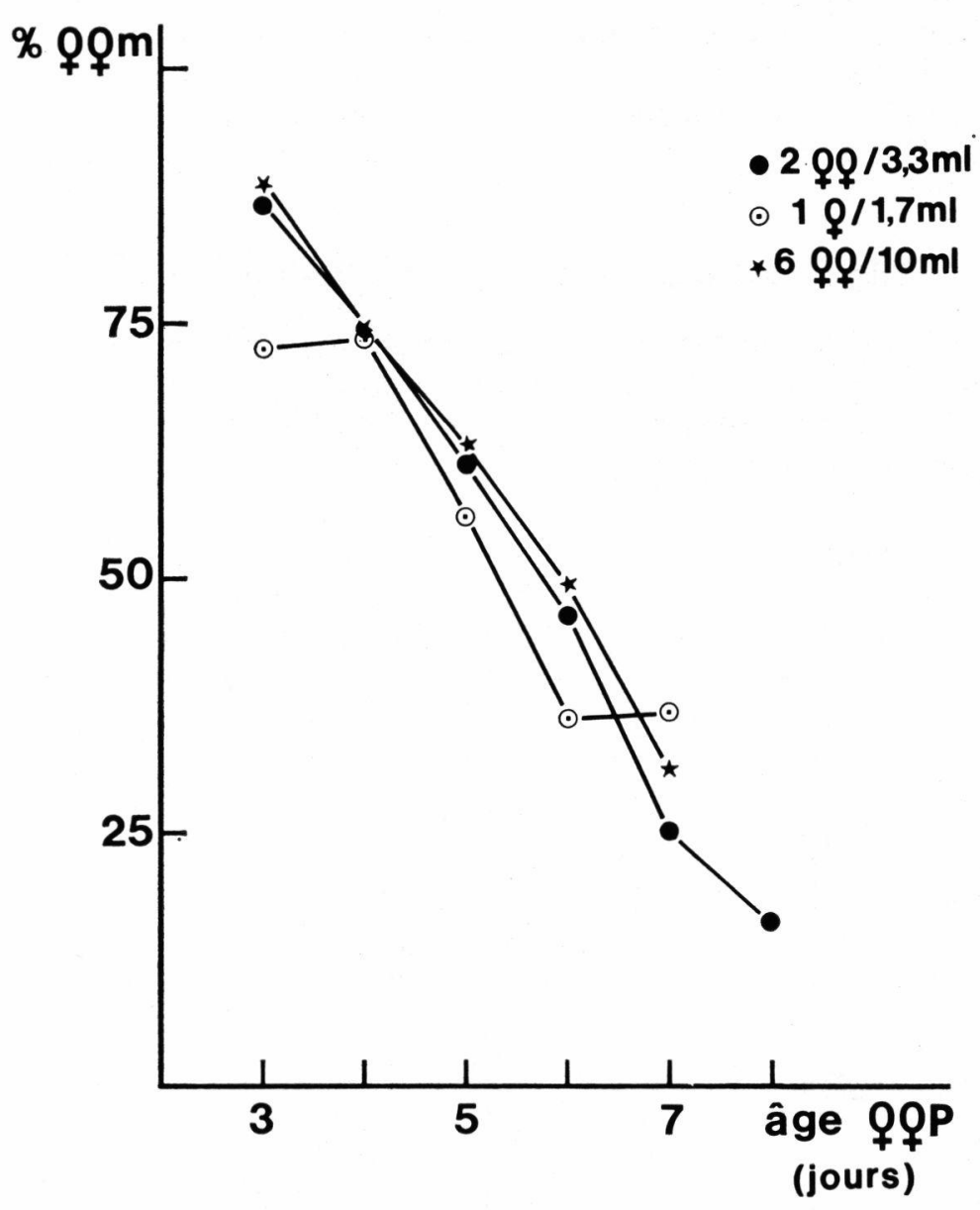

Fig. 2. - Evolution du taux de femelles mictiques en fonction de l'âge de la mère à la densité $0,6 \% / \mathrm{ml}$, pour trois groupements différents.

L'âge des femelles parentales exerce une influence significative dans tous les cas. Ceci confirme nos résultats antérieurs (Pourriot et Rougier, 1976 ; Rougier and al., 1977). Les courbes (fig. 1 à 4) représentant l'évolution des pourcentages de femelles mictiques en fonction de l'âge, ont toutes une forme similaire: les taux de femelles mictiques décroissent linéairement avec l'âge des parents. Cette décroissance s'observe en général dès le début de la ponte, à l'exception des courbes obtenues à partir de 1 et 2 femelles parentales à la densité 2 (fig. 4), où les taux de femelles mictiques ne diminuent qu'à partir du $3^{\mathrm{e}}$ jour de ponte $\left(=5^{\mathrm{e}}\right.$ jour de vie de la mère $)$. 


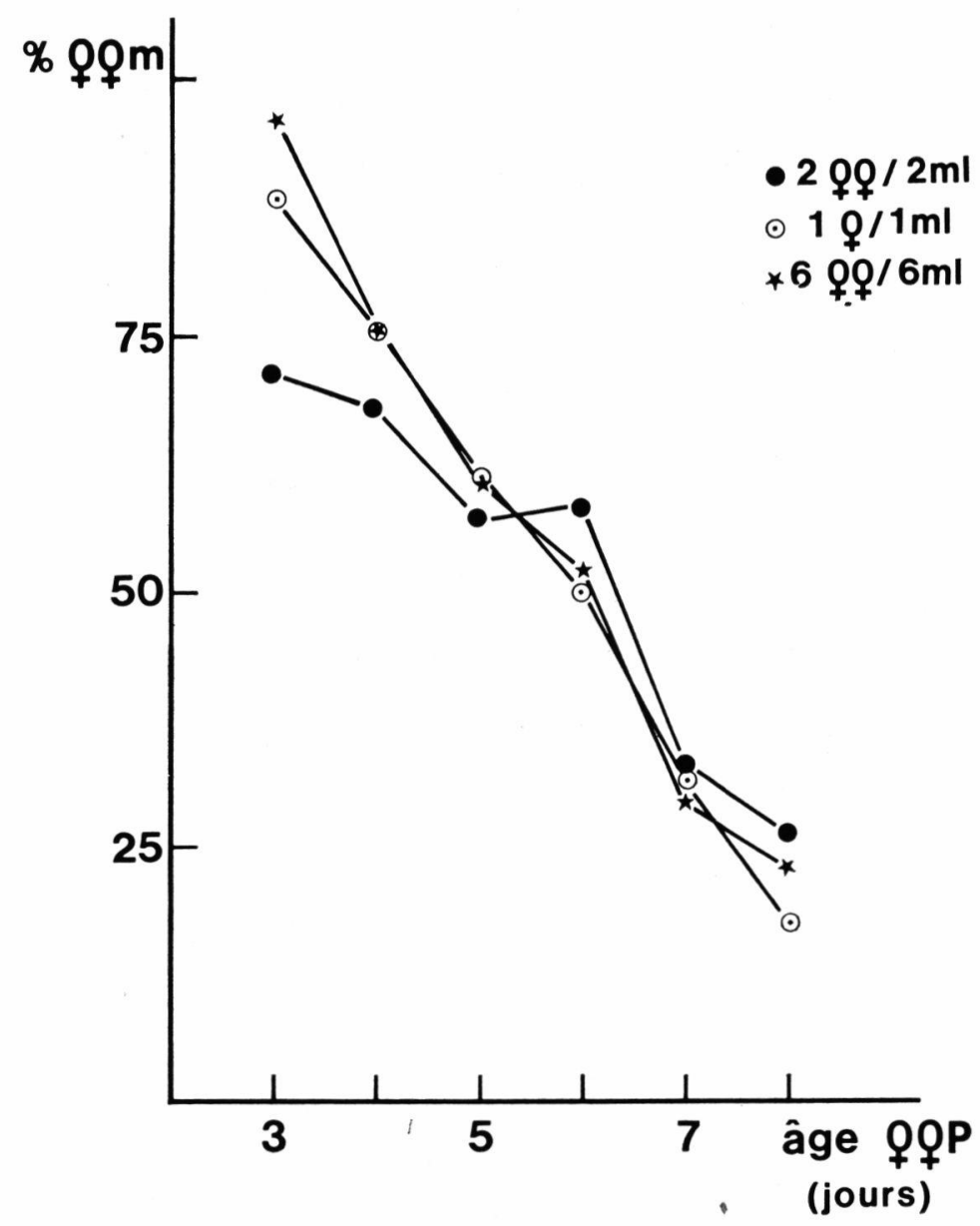

FIg. 3. - Evolution du taux de femelles mictiques en fonction de l'âge de la mère à la densité $1 \% / \mathrm{ml}$, pour trois groupements différents.

L'influence du groupement existe mais pas constamment. Aucun effet de groupement n'apparaît aux densités moyennes $(0,6$ et $1 \% / \mathrm{ml}$ ) : les 3 courbes (fig. 2 et 3 ) sont confondues. Par contre, aux densités extrêmes $(0,2$ et $2 \% \% / \mathrm{ml})$, le groupement exerce une influence significative (tabl. 1) mais inverse selon le groupement considéré: les taux de femelles mictiques sont supérieurs lorsque les femelles parentales sont groupées par 2 et inférieurs lorsque ces femelles sont groupées par 6 (par rapport aux taux produits par les femelles isolées; fig. 1 et 4 ). 


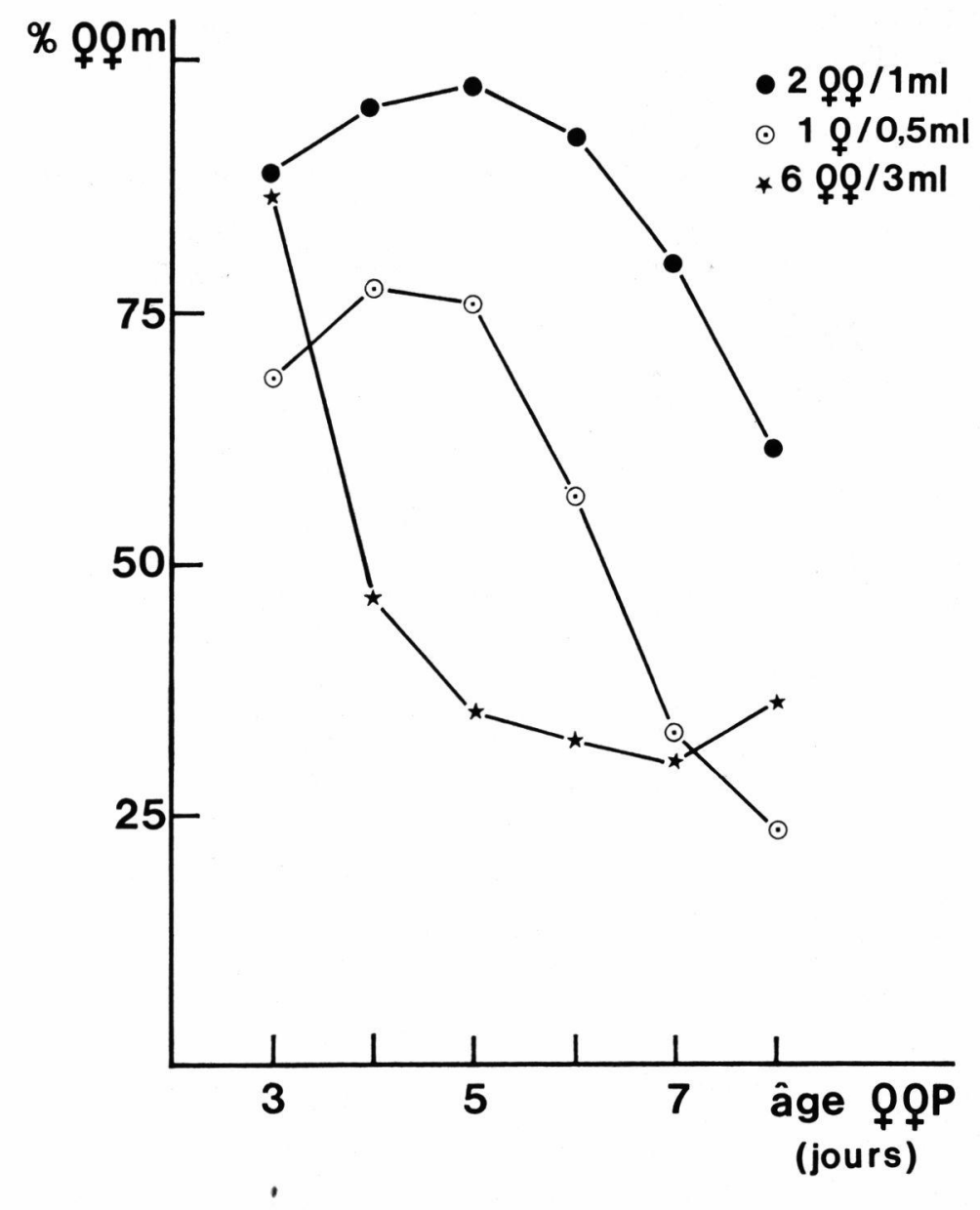

Fig. 4. - Evolution du taux de femelles mictiques en fonction de l'âge de la mère à la densité $2 \% \% / \mathrm{ml}$, pour trois groupements différents.

A ces deux densités extrêmes, l'analyse des groupements, comparés cette fois 2 à 2 (tabl. 2), montre que les réponses fournies par les femelles isolées et les femelles groupées par deux sont significativement différentes. Il en est de même pour les taux produits par les femelles groupées par deux et par les femelles groupées par six. Mais il n'apparaît pas de différences significatives entre les pourcentages quotidiens fournis par les femelles isolées et ceux fournis par les femelles groupées par six. Cependant, à la densité la plus élevée $(2 \mathrm{q} / \mathrm{ml})$, on relève des différences significatives entre les groupements 1 et 6 pour la période allant du $4^{\mathrm{e}}$ au $7^{\mathrm{e}}$ jour de la vie des 
(7)

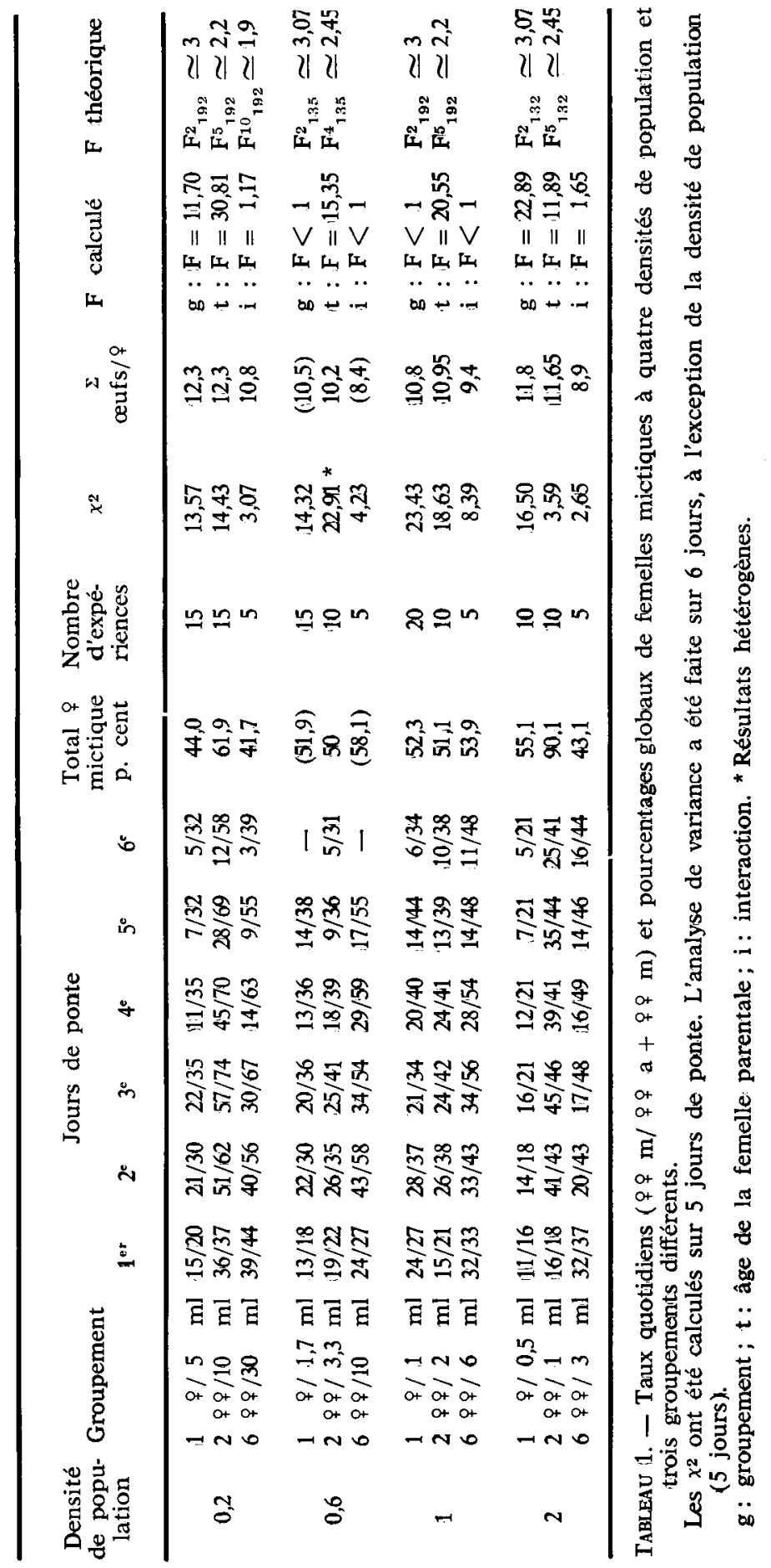


femelles parentales $\left(F=6,79\right.$ pour $F^{1}{ }_{24}=4,26$ ), c'est-à-dire en faisant abstraction des $3^{\mathrm{e}}$ et $8^{\mathrm{e}}$ jours de ponte (points extrêmes des courbes de la figure 4) où les relations entre les pourcentages sont inversées.

\begin{tabular}{|c|c|c|c|c|}
\hline $\begin{array}{l}\text { Densité de } \\
\text { population }\end{array}$ & $\begin{array}{c}\text { Groupements comparés } \\
(\mathrm{n} / \mathrm{v})\end{array}$ & F calculé & $F$ théorique & Effet observé \\
\hline 0,2 & $\begin{array}{l}1 / 5-2 / 10 \\
1 / 5-6 / 30 \\
2 / 10-6 / 30\end{array}$ & $\begin{array}{l}16,4 \\
<1 \\
24 ; 1\end{array}$ & $\begin{array}{l}F_{168}=3,9 \\
F_{108}=3,94\end{array}$ & $\begin{array}{l}+ \\
0 \\
-\end{array}$ \\
\hline 0,6 & 3 comparaisons & $<1$ & & $\mathbf{0}$ \\
\hline 1 & 3 comparaisons & $<1$ & & 0 \\
\hline 2 & $\begin{array}{l}1 / 0,5-2 / 1 \\
1 / 0,5-6 / 3 \\
2 / 1-6 / 3\end{array}$ & $\begin{array}{r}25,8 \\
1,4 \\
65,1\end{array}$ & $\begin{array}{l}F_{108}=3,94 \\
F^{1}{ }_{78} \simeq 4\end{array}$ & $\begin{array}{l}+ \\
0 \\
-\end{array}$ \\
\hline
\end{tabular}

Tableau 2. - Effets comparés du groupement sur le taux de femelles mictiques à 4 densités de population. Les valeurs de $F$ résultent d'une analyse de variance à 2 facteurs (groupement et temps) avec répétitions. Les valeurs concernant le $2^{\mathrm{e}}$ facteur (temps), toujours significatives n'ont pas été reportées dans le tableau.

L'influence du groupement sur la fécondité a été également testée. A la dentité 0,2 l'effet de groupement sur le nombre total de descendants produits en 6 jours par une femelle est nul $\left(F=1,8\right.$ pour $F_{32}^{2}$ $=3,32$ ). A la densité 2 , le nombre de descendants produits par les femelles groupées par 6 est significativement inférieur à celui fourni par les femelles isolées $\left(F=7,57\right.$ pour $\left.F^{13}=4,67\right)$ alors qu'il n'existe pas de différences significatives quant au nombre de descendants entre les femelles isolées et les femelles groupées par $2(F<0,01$ pour $\mathrm{F}^{1}{ }_{18}=4,41$ ).

Cet effet négatif du groupement par 6 sur la fécondité est confirmé par une analyse de variance à deux facteurs (groupement et âge des femelles) avec répétitions (tabl. 3) portant sur les taux quotidiens

\begin{tabular}{ccccl}
\hline $\begin{array}{c}\text { Densité } \\
\text { de population }\end{array}$ & $\begin{array}{c}\text { Groupements } \\
\mathrm{n} / \mathrm{v}, \text { comparés }\end{array}$ & $\mathrm{F}$ calculé & $\mathrm{F}$ théorique & \multicolumn{1}{c}{ Effet } \\
\hline \multirow{2}{*}{0,2} & $1 / 5-2 / 10$ & 0,02 & $\mathrm{~F}^{1}{ }_{168}=3,90$ & nul \\
& $1 / 5-6 / 30$ & 10,13 & & significatif - \\
2 & $1 / 0,5-2 / 1$ & 0,05 & $\mathrm{~F}^{1}{ }_{108}=3,92$ & nul \\
& $1 / 0,5-6 / 3$ & 29,57 & & significatif - \\
\hline
\end{tabular}

TABlEAU 3. - Résultats de l'analyse de variance concernant l'influence du groupement sur la fécondité (nombre de descendants par jour par femelle, pendant 6 jours). 
de femelles mictiques (fig. 5 et 6). Les nombre de jeunes produits par jour par une femelle parentale n'est pas significativement différent entre les femelles groupées par 2 et les femelles isolées (densité $0,2: F=0,02$ pour $\mathrm{F}^{1}{ }_{168}=3,90$; densité $2: \mathrm{F}=0,05$ pour $\mathrm{F}^{1}{ }_{108}=3,92$ ). Mais entre les femelles groupées par 6 et les femelles isolées, ces différences sont significatives aussi bien à la densité $0,2 \quad(F=10,13$ pour $\left.\mathrm{F}^{1}{ }_{168}=3,90\right)$ qu'à la densité $2\left(\mathrm{~F}=29,57\right.$ pour $\left.\mathrm{F}^{1}{ }_{108}=3,92\right)$.

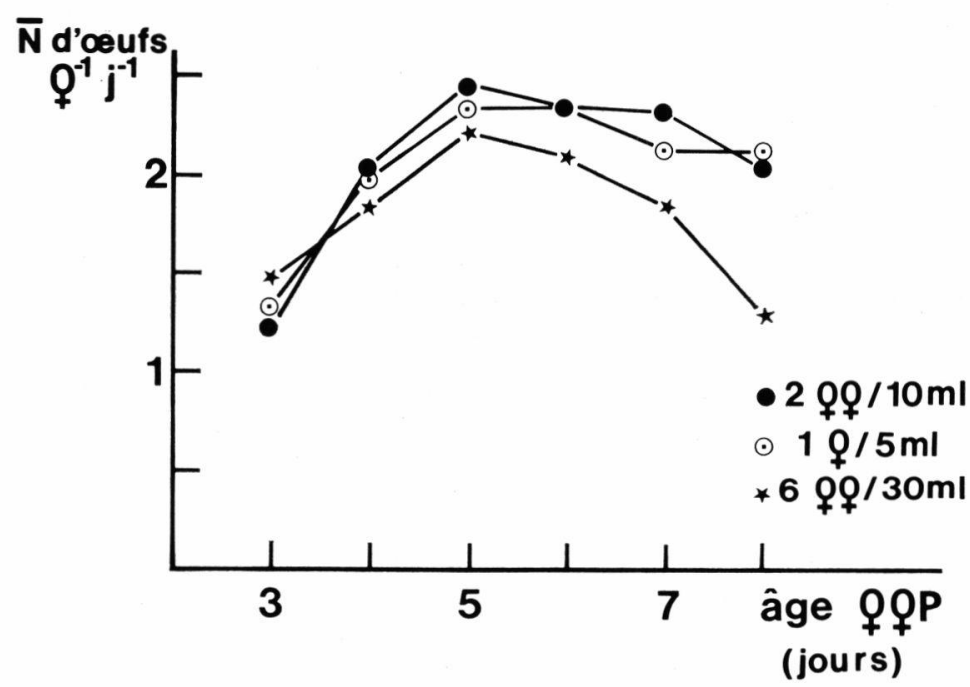

FIG. 5. - Evolution de la fécondité quotidienne à la densité de population $0,2 \% / \mathrm{ml}$, pour trois groupements différents.

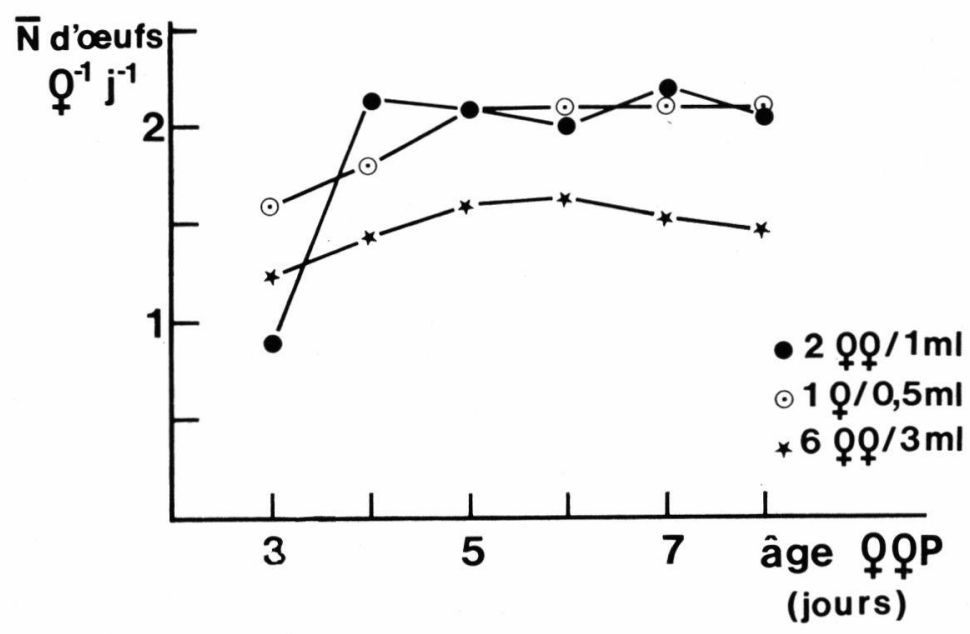

Fig. 6. - Evolution de la fécondité quotidienne à la densité $2 \% \% / \mathrm{ml}$ pour trois groupements différents. 
Remarque: la courbe obtenue à la densité de 1 ind/0,5 $\mathrm{ml}$ (fig. 4) résulte de deux expériences effectuées à 2 ans d'intervalle environ: mai 1974 et mars 1976 . Un calcul de $\chi^{2}$ montre que les pourcentages quotidiens fournis par chacune des femelles parentales des deux séries sont homogènes. Cette reproductibilité des résultats à deux ans d'intervalle confirme la stabilité génétique du clone expérimenté. On peut aussi en conclure que l'évolution des pourcentages quotidiens (courbe 1/0,5 de la fig. 4) n'est pas due au hasard.

\section{2. - Effets de la densité de population dans 3 groupements différents}

$\mathrm{Si}$, à l'inverse de ce qui précède, on considère les effets de la densité, pour chaque groupement, sur l'évolution des pourcentages quotidiens de femelles mictiques, on constate que ces effets varient d'un groupement à l'autre (tabl. 4). Par exemple, les différences entre les densités extrêmes $(0,2$ et $2 \% / \mathrm{ml})$ sont significatives lorsque les femelles sont isolées (seule différence significative à ce groupement : $\mathrm{F}=4,39$ pour $\left.\mathrm{F}^{1}{ }_{190}=3,9\right)$ et lorsqu'elles sont groupées par $2(\mathrm{~F}=$ 35,4 pour $\left.\mathrm{F}^{1}{ }_{109}=3,9\right)$. Mais dans le groupement par 6 , il n'apparaît aucune différence significative entre ces deux densités $(F=0,07$ pour $\left.\mathrm{F}_{90}^{1}=4\right)$.

\begin{tabular}{ccccccccccc}
\hline & \multicolumn{2}{c}{ Groupement } & par 11 & \multicolumn{2}{c}{ Groupement } & par 2 & \multicolumn{2}{c}{ Groupement par 6} \\
\hline Densités & 0,6 & 1 & 2 & 0,6 & 1 & 2 & 0,6 & 1 & 2 \\
de population & & & & & & & & & \\
$\mathbf{0 , 2}$ & 0,07 & 2,89 & $4,39^{*}$ & $24,2^{*}$ & $15,1^{*}$ & $35,4^{*}$ & $18,6^{*}$ & $23,4^{*}$ & 0,07 \\
$\mathbf{0 , 6}$ & & 1,92 & 3,14 & & 0,62 & $107^{*}$ & & 0,65 & $12,4^{*}$ \\
1 & & & 0,16 & & & $90^{*}$ & & & $16,7^{*}$ \\
\hline
\end{tabular}

TABlfau 4. - Valeurs de $F$ résultant d'une analyse de variance à 2 facteurs (densité de population et temps) avec répétitions, pour 3 groupements différents. Les valeurs correspondant au $2^{c}$ facteur (temps) sont toutes significatives et n'ont pas été reportées dans le tableau. Les densités de population sont comparées 2 à 2 .

$\approx$ : valeurs significatives.

Quel que soit le groupement, les réponses fournies à la densité 0,6 et celles obtenues à la densité 1 ne diffèrent pas non plus significativement.

\section{3. - DISCUSSION}

Comme présumé, puisqu'il s'agit d'un phénomène endogène, l'influence de l'âge des femelles parentales s'exerce en permanence quels que soient la densité de population et le groupement. L'âge 
des femelles parentales est donc le principal facteur contrôlant le taux de femelles mictiques produites en $F_{1}$, dans les conditions expérimentales précédemment définies. Ce taux n'étant jamais nul, la densité de population ne peut pas être considérée comme un facteur "inducteur", caractérisant une réponse du type " tout ou rien", pas plus d'ailleurs que dans les expériences de Gilbert (1963) où les taux de femelles mictiques sont plus souvent faibles que nuls, aux basses densités de population.

L'interprétation des observations concernant l'influence de la densité de population et du groupement est assez délicate du fait de l'inconstance de cette influence.

Bien que l'analyse de variance portant sur les pourcentages globaux ait mis en évidence une influence de la densité de population, les analyses plus détaillées concernant les taux quotidiens de femelles mictiques ne permettent pas d'aller au-delà de la simple constatation des faits.

Ceux-ci s'ordonnent mieux lorsque l'on considère non plus la densité de population mais le groupement. Aux densités 0,2 et 2, le groupement par 2 a un effet nettement favorable. Cet effet du groupement, similaire à celui observé chez Notommata copeus (Clément et Pourriot, 1975) pourrait s'interpréter également par la sécrétion dans le milieu, par une femelle parentale, d'une substance $\left(\mathrm{AF}_{\mathrm{m}}\right)$ agissant sur les autres femelles en augmentant le taux de femelles mictiques de leur descendance mais ne modifiant pas leur taux de reproduction (cf. tabl. 3). Chaque femelle serait plus sensible aux substances sécrétées par les autres femelles qu'à ses propres sécrétions.

L'influence du groupement par 6 est bien différente et plus difficile à interpréter. Ses effets, défavorables, s'exercent à la fois sur le taux de femelles mictiques et sur la fécondité.

A la plus forte densité de population $(2 \% / \mathrm{ml})$ on peut supposer que l'effet favorable apparent (mais non significatif) du groupement au début de la ponte est rapidement masqué par un effet défavorable se surimposant dès le $2^{e}$ jour de ponte. Cet effet défavorable pourrait être dû à un excès de concentration de la substance $A_{\mathrm{m}}$ à condition d'admettre soit qu'elle est sécrétée par chaque femelle à un taux (ou à une vitesse) d'autant plus élevé que le nombre de femelles en présence est plus grand, soit que chaque femelle devient d'autant plus sensible (synergisme). Cette hypothèse implique l'existence d'un seuil de concentration de la substance $\mathrm{AF}_{\mathrm{m}}$ déterminant une inversion de son action physiologique (phénomène observé pour de nombreuses substances physiologiquement actives). L'excrétion d'une substance différente, ayant une action néfaste sur le métabolisme $\left(\mathrm{DF}_{\mathrm{m}}\right.$ ?), assujétie des mêmes conditions, pourrait aussi rendre compte de cet effet défavorable. 
Pour expliquer la disparition de ces effets, tant favorables que défavorables, du groupement aux densités moins élevées $(0,6$ et 1$)$ et leur réapparition à la densité la plus faible $(0,2), 10$ fois inférieure à la plus forte (2), on peut admettre une variation cyclique de l'influence du groupement en fonction de la densité de population.

S'il s'avère indéniable que la densité de population et le groupement exercent une influence sur la production de femelles mictiques, celle-ci ne paraît pas simple ainsi qu'en témoigne la complexité des hypothèses avancées (qui restent purement spéculatives puisque non testées).

\section{Comparaison aVEC les RÉSUltats DE GILbERT (1963)}

Dans son travail publié en 1963, Gilbert a suivi la descendance de femelles isolées ou groupées par 6 à deux densités de population: $0,66 \% / \mathrm{ml}$ et $4 \% / \mathrm{ml}$. Gilbert observe un effet prépondérant de la densité de population: le taux de femelles mictiques produites à densité élevée est 2,5 à 3 fois supérieur à celui obtenu à faible densité, quel que soit le groupement. Bien que les différences ne soient pas significatives, les pourcentages moyens de femelles mictiques sont légèrement plus élevés lorsque les femelles sont groupées par 6. Ces résultats diffèrent en tous points de ceux que nous avons obtenus avec cette même espèce : un effet globalement positif et significatif de la densité de population (mais moins important que celui observé par Gilbert) variable selon les groupements; un effet négatif et significatif du groupement par 6 (tabl. 5). De plus, bien que nous n'ayons pas expérimenté à la densité la plus forte testée par Gilbert $(4 \% \% / \mathrm{ml})$, les taux de femelles mictiques obtenus pour la totalité du temps d'expérience sont bien plus élevés que ceux notés par Gilbert (de 41,7 à $90,1 \%$ pour $B$. calyciflorus GC, de 0 à $50 \%$ pour la souche de Gilbert) pour un nombre de descendants par femelle voisin ( 9 à 12 et 13 ).

\begin{tabular}{|c|c|c|c|c|c|c|c|}
\hline & & $\mathbf{T}$ & 'Nourriture & $\begin{array}{l}\text { Effet } \\
\text { de } \\
\text { l'âge }\end{array}$ & $\begin{array}{c}\text { Effet } \\
\text { de la densité }\end{array}$ & $\begin{array}{l}\text { Effet du } \\
\text { groupement } \\
\text { par } 2\end{array}$ & $\begin{array}{l}\text { Effet du } \\
\text { groupement } \\
\quad \text { par } 6\end{array}$ \\
\hline \multirow{2}{*}{ : } & Gilbert & $25^{\circ}$ & $\begin{array}{l}\text { Euglena } \\
\text { gracilis }\end{array}$ & $\begin{array}{l}\text { non } \\
\text { testé }\end{array}$ & $\begin{array}{c}++ \\
\text { indépendant } \\
\text { du } \\
\text { groupement }\end{array}$ & non testé & $\begin{array}{c}\text { non } \\
\text { significatif }\end{array}$ \\
\hline & GC & $15^{\circ}$ & $\begin{array}{l}\text { Phacus } \\
\text { pyrum }\end{array}$ & ++ & $\begin{array}{c}+ \\
\text { fonction } \\
\text { du } \\
\text { groupement }\end{array}$ & $\begin{array}{l}\stackrel{+}{\text { fonction de }} \\
\text { la densité }\end{array}$ & $\begin{array}{l}\text { fonction de } \\
\text { la densité }\end{array}$ \\
\hline
\end{tabular}

Tableau 5. - Comparaison des conditions expérimentales et des réponses fournies par la souche de Gilbert (1963) et par la souche GC. 
Outre l'origine de la souche de $B$. calyciflorus, la principale différence entre les conditions expérimentales de Gilbert et les nôtres réside dans la température $\left(25^{\circ}\right.$ au lieu de $\left.15^{\circ}\right)$. La qualité de la nourriture diffère également (Euglena gracilis au lieu de Phacus pyrum) quoique ces flagellés soient tous deux des Eugléniens.

Il faut donc aclmettre que les conditions de température et de qualité de la nourriture modifient les réponses aux facteurs densité de population et groupement ou (et) que les deux souches sont génétiquement différentes.

\section{TRAVAUX CITES}

BuCHNER (H.). 1941. - Experimentelle Untersuchungen über den Generationwechsel der Rädertiere. II. Zool. Jb. Physiol., 60, 3, 279-344.

Buchner (H.), Kiechle (H.), TIEFenbacher (L.). 1969. - Untersuchungen über die Bedingungen der heterogonen Fortpflanzungsarten bei den Rädertiere. I. Zool. Jb. Physiol., 74, 329-426.

Clément (P.) et Pourriot (R.). 1975. - Influences du groupement et de la densité de population sur le cycle de reproduotion de Notommata copeus (Rotifère). I. Arch. Zool. exp. gén., 1116, 375-422.

Gilbert (J. J.). 1963. - Mictic female production in the Rotifer Brachionus calyciflorus. J. exp. Zool., 153, 2, 113-124.

KING (C. E.). 1977. -- Genetics of reproduction, variation and adaptation in Rotifers. Arch. Hydrobiol. Beih., Ergebn. Limnol., 8, 187-201.

Pourriot (R.) et Rougier (C.). 1976. - Influence de l'âge des parents sur la production de femelles mictiques chez Brachionus calyciflorus (Pallas) et $B$. rubens Ehr. (Rotifères). C. R. Acad. Sci., 283 D, 1497-1500.

Rougier (C.) et Pourriot (R.). 1977. - Aging and control of the reproduction in Brachionus calyciflorus (Pallas) (Rotatoria). Exp. Gerontol., 9, 137-151.

Rougier (C.), Pourriơ (R.) et Clément (P.). 1977. - Determination of mixis in Brachionus. Arch. Hydrobiol. Beih., Ergeln. Limnol., 8, 163-166. 